\title{
Procalcitonin implication in renal cell apoptosis induced by acute pyelonephritis in children
}

\author{
Hafid Belhadj-Tahar ${ }^{1,2}$ \\ Yvon Coulais ${ }^{2}$ \\ Mathieu Tafani \\ François Bouissou ${ }^{3}$ \\ 'Groupe Santé Recherche, Toulouse, \\ France; ${ }^{2}$ EA 3033 Université Paul \\ Sabatier III, Toulouse, France; ${ }^{3}$ Service \\ de Néphrologie Pédiatrique, $\mathrm{CHU}$ \\ Purpan, Toulouse, France
}

\begin{abstract}
The aim of this biomedical trial was to clarify the physiological role of procalcitonin (PCT) in renal parenchyma apoptosis and fibrosis caused by acute childhood pyelonephritis. This prospective study enrolled 183 children. All children were treated with bi-therapy according to the French consensus on acute pyelonephritis treatment dated November 16, 1990: intravascular administration of ceftriaxone $50 \mathrm{mg} / \mathrm{kg}$ /day and netromicine $7 \mathrm{mg} / \mathrm{kg} /$ day during the first 48 hours, followed by specific antibiotherapy suited to antibiogram. On admission, PCT, C-reactive protein, and phospholipase A2 were quantified in serum. Scintigraphy monitoring with ${ }^{99 \mathrm{~m} T c-D M S A}$ was performed on day 4 and 9 months later, in the presence of persistent abnormalities. On day 4, 78\% presented renal parenchyma alterations and 30\% renal fibrosis 9 months after admission. Paradoxically, PCT level was significantly lower in the presence of renal fibrosis due to cell apoptosis (4.19 vs $\left.7.59 \mu \mathrm{gL}^{-1}\right)$. A significant increase in PCT indicated favorable progress (recovery 7.55 vs aggravation 3.34 ) and no difference between recovery and improvement. This result suggests the protective effect of PCT against apoptosis by nitric oxide down-regulation.
\end{abstract}

Keywords: acute pyelonephritis, procalcitonin, apoptosis, fibrosis, technetium-DMSA

\section{Background}

Researchers generally agree that increased procalcitonin (PCT) synthesis is detrimental to the host because a high concentration of this calcitonine precursor reflects the severity of sepsis and is predictive of mortality (Bossink et al 1999). Procalcitonin, a 116 amino acid peptide, is produced physiologically by thyroid C-cells and cleaved by specific protease. This prohormone is found at low concentration $(<0.1 \mathrm{ng} / \mathrm{mL})$ in healthy subjects and at high concentration in critically ill patients with a particular infection or shock. Although the role and production site of the PCT are unclear, this peptide induced by tumor necrosis factor alpha (TNF- $\alpha$ ) is currently considered to be an effective inflammatory marker used to monitor septic shock, pancreatic necrosis, or burn injuries (Venet et al 2002).

Recently, our research has focused on how PCT is implicated in acute childhood pyelonephritis (AP), followed in $30 \%$ of cases by renal fibrosis (RF) leading to chronic sequelae (such arterial hypertension gravidic toxemy). Renal fibrosis results from functional parenchyma disorder consecutive to renal cell apotosis, usually mediated by lipopolysaccharide (LPS)-induced TNF- $\alpha$ production. During the infection of the renal parenchyma, the bacterial LPS of the membranes induces the release of TNF, interleukin-1 (IL1), and IL6 (Oberhoffer et al 1999).

Pro-inflammatory TNF- $\alpha$ activates nitric oxide (NO) release, which is catalyzed by nitric oxide synthase (NOS) in the presence of $\mathrm{Ca}^{2+} /$ calmodulin. Thus intracellular calcium concentration is crucial for $\mathrm{Ca}^{2+} /$ calmodulin complex formation and NOS activation-inducing apoptosis (Arena et al 2002).
Correspondence: Hafid Belhadj-Tahar Groupe Santé Recherche, 35 Bernard de Ventadour, 31300 Toulouse (France) Tel +33 561492030

$\mathrm{Fax}+33561492040$

Email belhadj.h@chu-toulouse.fr 
In fact, cell viability and apoptosis depends on the spatiotemporal organization of cytosolic $\mathrm{Ca}^{++}$leading to transmembrane polarization magnitude (Lang et al 2005). In this context, the renal scintigraphy using the polar ${ }^{99 m}$ Tc-DMSA complex allows parenchyma exploration and then monitoring of acute and chronic lesions due to membrane adsorption attenuation (Belhadj-Tahar et al 2000).

The aim of this work is to clarify the physiological role of PCT in AP by monitoring the early and later renal lesions (edema/fibrosis) using ${ }^{99 \mathrm{~m} T c-D M S A}$ parenchyma exploration.

\section{Material and methods}

This prospective study enrolled 183 children. All subjects exhibited clinical and biological infectious syndrome, with abdominal or lumbar pains and positive urine culture before antibiotherapy administration. All children were treated with bi-therapy according to the French consensus on acute pyelonephritis treatment dated November 16, 1990: intravascular administration of ceftriaxone $50 \mathrm{mg} / \mathrm{kg}$ /day and netromicine $7 \mathrm{mg} / \mathrm{kg}$ /day during the first 48 hours, followed by specific antibiothérapy suited to antibiogram.

On admission C-reactive protein (CRP), phospholipase $A_{2}$, and procalcitonin were measured by the following methods: CRP turbidimetric immunoassay (Olympus diagnostica, $\mathrm{GmbH}$ ) $\mathrm{PLA}_{2}$ : fluometric assay (SFM 25 Kontron Instruments) and PCT: luminescent immunoassay (Brahms diagnostica $\mathrm{GmbH}$ ). Procalcitonin in serum were quantified as well as CRP and phospholipase $\mathrm{A}_{2}$. Scintigraphy monitoring with intravascular ${ }^{99 \mathrm{~m}} \mathrm{Tc}$-DMSA (CIS Bio International, France) was performed according to MacKenzie protocol (MacKenzie 1996) on day 4 and nine months after admission in the presence of persistent abnormalities.

The scintigraphic parenchyma lesions were classified into 4 categories: absence (stage 0 ), unique and focal (stage II), multiple but unilateral (stage II), and bilateral (stage III).

\section{Results}

At day 4, 78\% (107/136) had scintigraphic parenchyma renal alterations and 30\% (17/57) presented renal fibrosis at 9th month. On admission, except for CRP, both PLA 2 and PCT were significantly higher in patients with early scintigrahic alteration (PCT: 7.85 vs $2.36 \mu \mathrm{g} / \mathrm{L}$ and PLA $_{2}$ : 72.1 vs $29.9 \mathrm{IU} / \mathrm{L})$ (Table1).

\section{Discussion}

During the infection of the renal parenchyma, the bacterial LPS of the membranes induces the release of TNF, IL1, and IL6.

These cytokines are capable of evoking fever, inflammation syndrome, and cell apoptosis followed by fibrosis (Figure 1).

In the acute phase, IL1 and IL6 induce hepatic production of CRP and PLA 2 leukocyte release. PLA $\mathrm{P}_{2}$ is directly implied in the inflammatory process including the prostaglandins production from arachidonic acid in the presence of cyclooxygenase (Diaz 1996). Whereas, procalcitonin CRP and $\mathrm{PLA}_{2}$ have weak discriminative power for predictive renal fibrosis regarding to their AUC values below 0.65 (see Figure 1).

We hypothesized that PCT down-regulates nitric oxide (NO) release produced by NO-Synthase activated by TNF- $\alpha$ pro-inflammatory cytokine (Figure 2 ). Indeed, PCT was paradoxically significantly lower in the presence of chronic renal fibrosis $\left(4.19 \pm 1.03\right.$ vs $\left.7.59 \pm 3.45 \mu \mathrm{gL}^{-1}\right)$. We observe a significant PCT increase in the favorable progress (recovery $7.55 \pm 4.02$ vs aggravation $3.34 \pm 1.09$ ) and no difference between recovery and improvement evolution. These results suggest the protective effect of PCT against apoptosis resulting from down-regulation of nitric oxide release catalyzed by NO-synthase (Figure 1) and corroborate the previous In vitro observations (Hoffmann et al 2003).

Table I Characteristics of 107 patients with acute childhood pyelonephritis

\begin{tabular}{|c|c|c|c|c|c|c|}
\hline & lesion & age & Sex ratio & CRP $\left(\mathrm{mgL}^{-1}\right)$ & $\operatorname{PLA}_{2}\left(\right.$ IUL $\left.^{-1}\right)$ & PCT $\left(\mu \mathrm{gL}^{-1}\right)$ \\
\hline \multirow[t]{5}{*}{ 4th day } & Absence & $43.48 \pm 7.39 n=29$ & M: 7\%; F: I5\% & $77.16 \pm 10.21 \mathrm{n}=25$ & $29.90 \pm 7.83 n=27$ & $2.36 \pm 1.36 n=23$ \\
\hline & Stage $>0$ & $44.07 \pm 4.26 n=107$ & M: 29\%; F: 7I\% & $114.60 \pm 9.74 n=100$ & $72.03 \pm 7.94 n=87$ & $7.85 \pm 1.93 n=65$ \\
\hline & Stage I & $37.91 \pm 4.47 n=72$ & M: I5\%; F: 38\% & $114.60 \pm 9.0 n=66$ & $76.32 \pm 13.08 n=57$ & $6.40 \pm 1.99 n=47$ \\
\hline & Stage II & $48.70 \pm 7.30 n=21$ & M: 5\%; F: I0\% & $130.14 \pm 23.36 n=21$ & $32.70 \pm 6.72 n=17$ & $7.34 \pm 4.66 n=11$ \\
\hline & Stage III & $33.47 \pm 8.79 n=14$ & M: I\%; F: 9\% & $121.07 \pm 16.46 n=14$ & $104.65 \pm 28.47 n=13$ & $18.37 \pm 9.46 n=7$ \\
\hline \multirow[t]{2}{*}{ 9th month } & Absence & $39.86 \pm 6.78 n=40$ & M: I 7\%; F: 53\% & $101.16 \pm 12.20 n=37$ & $61.80 \pm 12.22 n=33$ & $7.59 \pm 3.45 n=24$ \\
\hline & Fibrosis (Stage I) & $27.19 \pm 5.54 n=17$ & M: 7\%; F: 23\% & $123.35 \pm 21.35 n=17$ & $81.65 \pm 20.38 n=13$ & $4.19 \pm 1.03 n=11$ \\
\hline
\end{tabular}



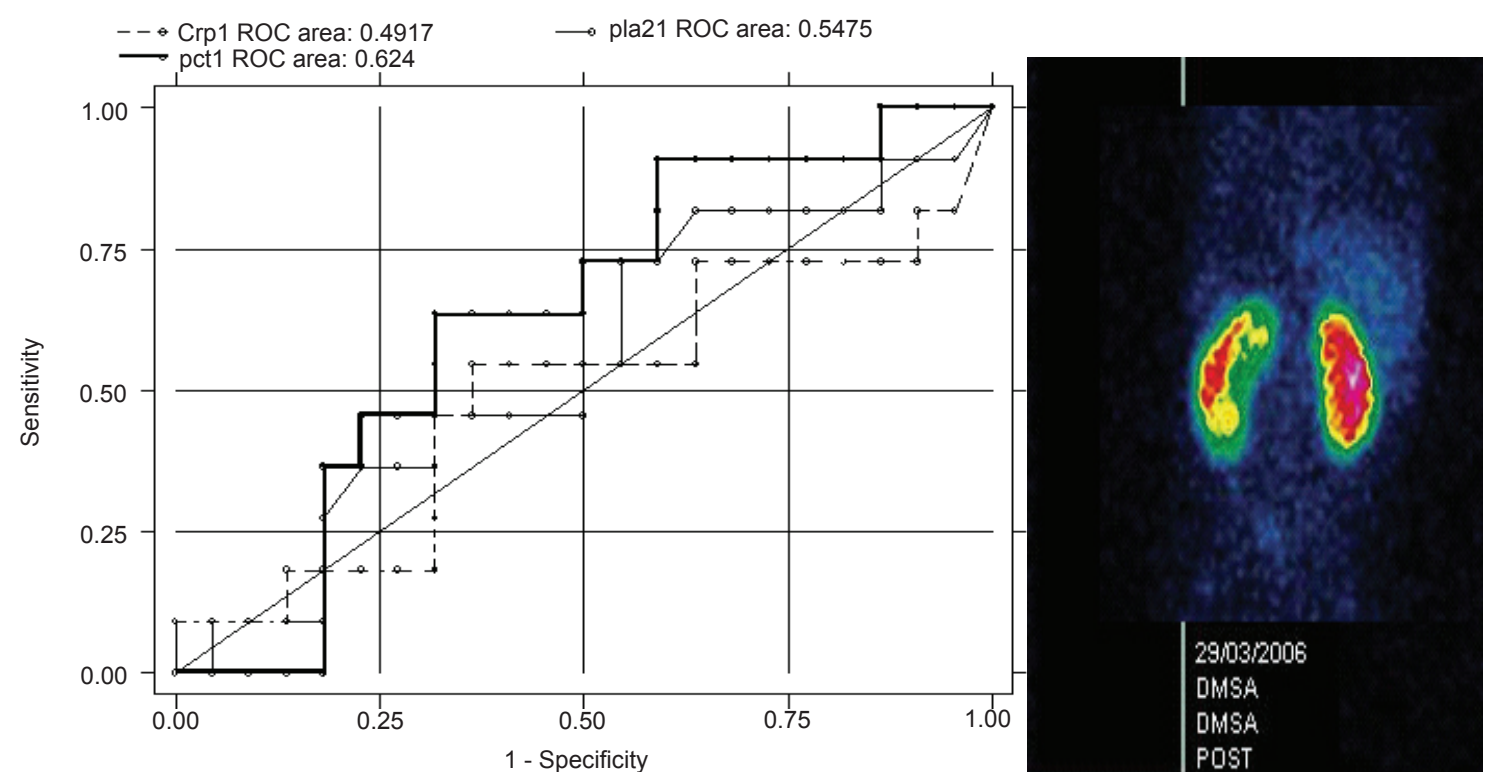

Figure I Receiver operating characteristics curves for C-reactive protein (CRP), phospholipase $\mathrm{A}_{2}$ (PLa $)$, procalcitonin (PCT), and renal fibrosis observed by ${ }^{99 \mathrm{~m} T c-D M S A}$ scintigraphy.

Note: Areas under curve (AUC) of the 3 markers were respectively (Cl: 0.26163-0.72I84) for CRP; 0.5475 (Cl: 0.33059-0.76445) for PLa and 0.6240 (Cl: $0.42373-0.8242$ I) for PCT.

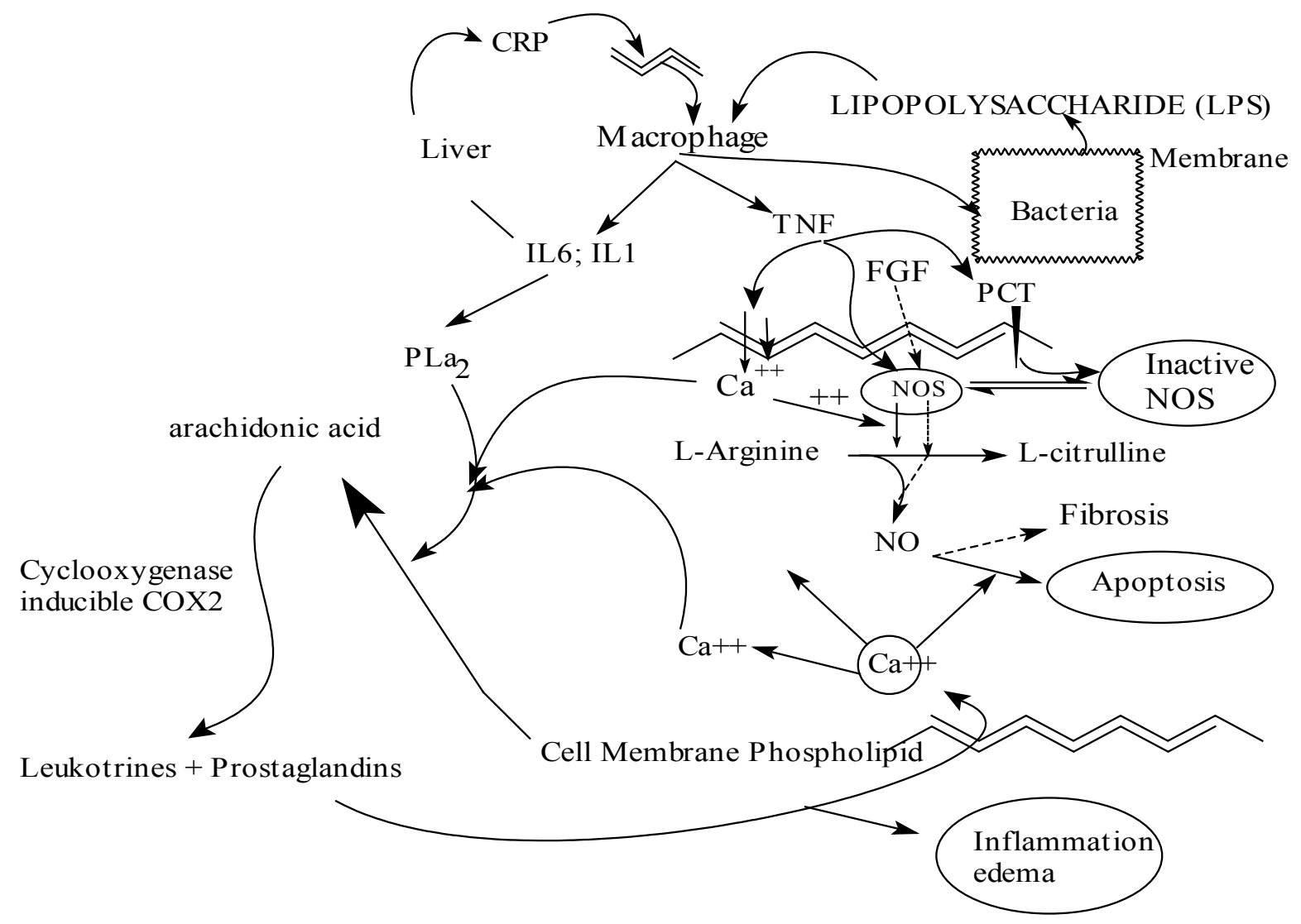

Figure 2 Physiopathology of pyelonephritis.

Abbreviations: IL, interleukin; NO, nitric oxide; NOS, NO synthase; PCT, procalcitonin; PLA, phospholipase $\mathrm{A}_{2}$; CRP, C-reactive protein; FGF, fibroblast growth factor; TNF, tumor necrosis factor. 
Table 2 Pyelonephetis progress according to procalcitonin concentration

\begin{tabular}{|c|c|c|c|}
\hline & No sequellae & Fibrosis sequellae & F. test \\
\hline \multirow[t]{2}{*}{ Early lesion stage I-II } & $7.59 \pm 3.45 \mathrm{mgL}^{-1} \mathrm{n}=24$ & $4.19 \pm 1.03 \mathrm{mgL}^{-1} \mathrm{n}=11$ & $\mathrm{P}<0.00 \mathrm{I}$ \\
\hline & Recovery stage $(0)$ & Aggravation stage $(\rightarrow I)$ & \\
\hline \multirow[t]{2}{*}{ Early lesion stage: I } & $7.55 \pm 4.02 \mathrm{mgL}^{-1} \mathrm{n}=20$ & $3.34 \pm 1.09 \mathrm{mgL}^{-1} \mathrm{n}=6$ & $\mathrm{p}<0.00 \mathrm{I}$ \\
\hline & recovery stage $(0)$ & Improvement stage $(\geq|| \rightarrow \mid)$ & \\
\hline Early lesion stage: $\geq$ II & $7.78 \pm 5.98 \mathrm{mgL}^{-1} \mathrm{n}=4$ & $4.60 \pm 2.36 \mathrm{mgL}^{-1} \mathrm{n}=5$ & ns \\
\hline
\end{tabular}

Note: The procalcitonin levels are listed according to pyelonephetis progress.

\section{Conclusion}

Our study shows that PCT plays key role in the renal parenchyma protection against cell apopotosis and fibrosis caused by acute pyelonephritis. this finding constitutes a original approach in the comprehension of apoptosis paradigm that causes parenchyma fibrosis.

\section{Acknowledgments}

The authors thank AFPreMed (Association Française de Promotion de la Recherche Médicale) for invaluable help. The authors report no conflicts of interest in this work.

\section{References}

Arena S, Pattarozzi A, Thellung V, et al. 2002. Nitric oxide production stimulated by the basic fibroblast growth factor requires the synthesis of ceramide. Ann NY Acad Sci, 973:94-104.

Belhadj-Tahar H, Darbieu MH, Boumahdi R. 2000. Conceptualisation of diagnostic agents : From empirical in vivo screening to rational in vitro predictive parameters. ATLA, 28:303-14.
Bossink AW, Groeneveld AB, Thijs LG. 1999. Prediction of microbial infection and mortality in medical patients with fever: plasma procalcitonin, neutrophilic elastase-alpha1-antitrypsin, and lactoferrin compared with clinical variables. Clin Infect Dis, 29:398-407.

Diaz BL, Arm JP. 2003. Phospholipase A(2). Prostaglandins Leukot Essent Fatty Acids, 69:87-97.

Hoffmann G, Totzke G, Seibel M, et al. 2001. In vitro modulation of inducible nitric oxide synthase gene expression and nitric oxide synthesis by procalcitonin. Crit Care Med, 29:112-6.

Lang F, Foller M, Lang KS, et al. 2005. Ion channels in cell proliferation and apoptotic cell death. J Membr Biol, 205:147-57.

MacKenzie JR. 1996. A review of renal scarring in children. Nucl Med Commun, 17:176-90.

Oberhoffer M, Karzai W, Meier-Hellmann A, et al. 1999. Sensitivity and specificity of various markers of inflammation for the prediction of tumor necrosis factor-alpha and interleukin-6 in patients with sepsis. Crit Care Med, 27:1814-8.

Venet C, Tardy B, Zeni F. 2002. [Marqueurs biologiques de l'infection en réanimation chez l'adulte: place de la procalcitonine.] Réanimation, 11:156-71. 